\title{
A case of pheochromocytoma with a marked decrease in catecholamine levels after rupture in which a good outcome was achieved by elective surgery
}

\author{
Norimitsu Murai1), Tetsushi Azami ${ }^{1)}$, Tatsuya Iida ${ }^{1)}$, Kentaro Mikura' ${ }^{1)}$, Hideyuki Imai ${ }^{1), 2)}$, Mariko Kaji ${ }^{1)}$, \\ Mai Hashizume ${ }^{1)}$, Yasuyoshi Kigawa ${ }^{1)}$, Go Koizumi ${ }^{1)}$, Rie Tadokoro' ${ }^{1)}$, Kei Endo' ${ }^{1)}$, Toru Iizaka ${ }^{1)}$, \\ Ryo Saiki ${ }^{1)}$, Fumiko Otsuka ${ }^{1)}$, Tomoko Norose ${ }^{2)}$, Motoki Yamagishi ${ }^{3)}$, Ippei Kurokawa ${ }^{3)}$, Nobuyuki Oike ${ }^{2)}$, \\ Haruaki Sasaki ${ }^{3)}$ and Shoichiro Nagasaka ${ }^{1)}$ \\ 1) Division of Diabetes, Metabolism and Endocrinology, Showa University Fujigaoka Hospital, Yokohama, Kanagawa 227-8501, \\ Japan \\ 2) Departments of Pathology, Showa University Fujigaoka Hospital, Yokohama, Kanagawa 227-8501, Japan \\ 3) Division of Urology, Showa University Fujigaoka Hospital, Yokohama, Kanagawa 227-8501, Japan
}

\begin{abstract}
Changes in imaging findings and hormone levels before and after pheochromocytoma rupture, as well as detailed histopathology of resected tumors, have rarely been reported. A 52-year-old woman developed hypertension and diabetes mellitus in 2014, but despite treatment with antihypertensive and hypoglycemic drugs, good control was not achieved. On April 2, 2016, the patient started to have headaches and palpitations, and on April 6, she visited our hospital. Plain computed tomography (CT) of the abdomen showed a 4-cm, isodense mass in the left adrenal gland, and the patient was hospitalized for further examination. Because the patient had hypertension, tachycardia, and hyperglycemia on admission, therapies for those were started. Catecholamine levels were markedly elevated. However, after the patient developed left flank pain on Day 4, antihypertensive and insulin therapies were no longer required. Plain CT then showed heterogeneous high density areas in the left adrenal mass. On Day 7, 3 meta-iodobenzylguanidine scintigraphy showed no abnormal uptake. On Day 8, contrast CT showed low density areas within the left adrenal tumor and contrast enhancement of the tumor margins, and catecholamine levels were markedly decreased. Elective left adrenal tumor resection was performed on Day 49. The capsule of the resected tumor was ruptured. Histopathology showed widespread hemorrhagic necrosis and viable cell components in the tumor margins. Positive chromogranin A staining of the tumor cells confirmed a diagnosis of pheochromocytoma. This patient displayed remarkable changes in imaging findings and hormone levels before and after pheochromocytoma rupture. Pheochromocytoma rupture and hemorrhagic necrosis were confirmed histopathologically.
\end{abstract}

Key words: Rupture of pheochromocytoma, Catecholamine levels, Hemorrhagic necrosis, Elective surgery

RUPTURED PHEOCHROMOCYTOMAS are rare, but elective surgery after systemic management often leads to favorable outcomes [1]. Changes in imaging findings and hormone levels before and after pheochromocytoma rupture, as well as detailed histopathology of resected tumors, have rarely been reported $[2,3]$.

Submitted Feb. 19, 2018; Accepted Jul. 4, 2018 as EJ18-0071 Released online in J-STAGE as advance publication Aug. 4, 2018 Correspondence to: Norimitsu Murai, MD, Division of Diabetes, Metabolism and Endocrinology, Showa University Fujigaoka Hospital, 1-30 Fujigaoka, Aoba-ku, Yokohama, Kanagawa 227-8501, Japan.

E-mail: uipnpzk2npq3@yahoo.co.jp
Atuk et al. [2] performed systemic management for pheochromocytoma and reported that, although a marked decrease in urinary catecholamine secretion was observed, and histopathology showed hemorrhagic necrosis of the tumor after shock occurred, no changes in imaging findings were noted. Maruyama et al. [3] reported changes both in serum and urinary catecholamine secretions, as well as changes in imaging findings, before and after pheochromocytoma rupture. However, histopathology showed no evidence of hemorrhagic necrosis of the tumor.

A patient who was initially treated conservatively for pheochromocytoma rupture in whom changes in imaging 

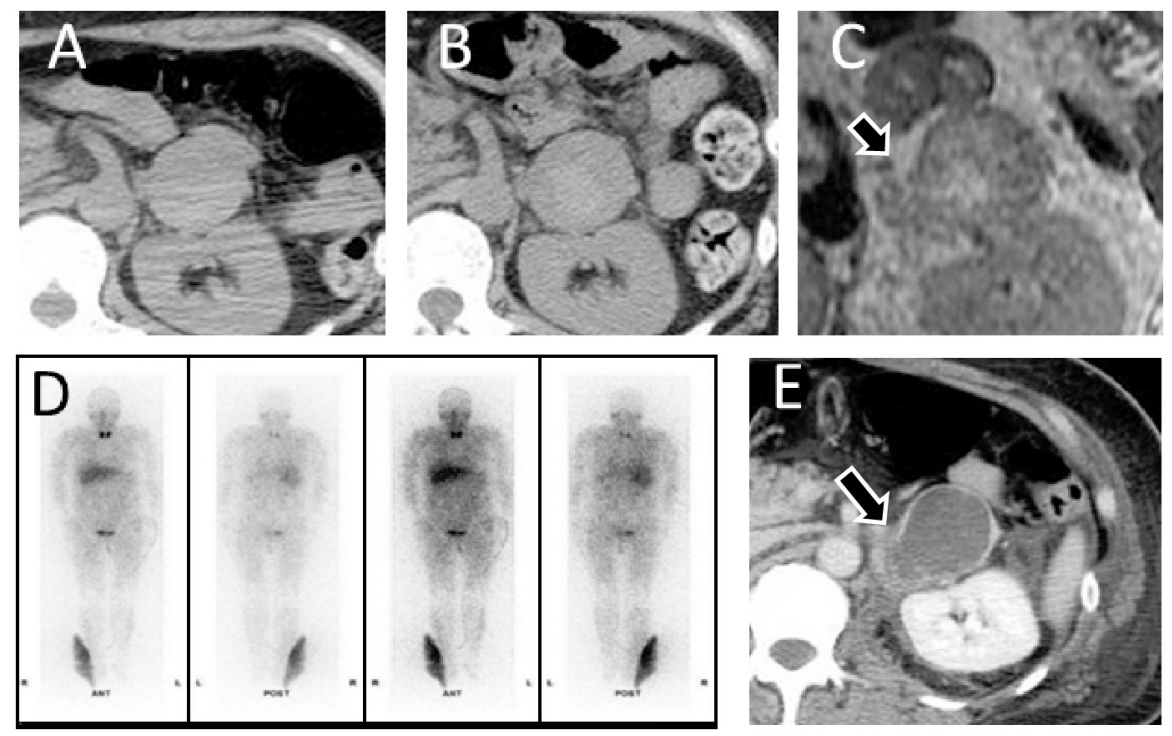

Fig. 1 Changes in imaging findings

A: Day 1 plain CT (tumor CT value: about $40 \mathrm{HU}$ ), B: Day 4 plain CT (tumor CT value: about 60 HU), C: Day 6 plain T2weighted MRI shows a heterogeneous mixture of high and low signal intensities in the tumor. Tumor border is indistinct (arrow), D: Day 7 MIBG scintigraphy shows no abnormal uptake, E: Day 8 contrast CT shows tumor rupture (arrow). CT, Computed tomography; HU, Hounsfield units; MRI, Magnetic resonance imaging; MIBG, 3 meta-iodobenzylguanidine.

findings and hormone levels were noted before and after rupture is described. Elective surgery was then performed for tumor resection, and histopathology showed hemorrhagic necrosis of the tumor.

\section{Case Presentation}

This patient was a 52-year-old woman who presented with a chief complaint of headache and palpitations. Regular medical examinations each year since 2011 had shown no abnormalities. However, during a medical examination in 2014, hypertension and diabetes mellitus were diagnosed, and she visited a local clinic. Oral drug treatment was started, but her blood pressure and glucose levels did not improve (blood pressure: about 160/90 $\mathrm{mmHg}$; heart rate: about 120/min; hemoglobin A1c (HbAlc): between $\geq 8 \%$ and $<9 \%$ ).

Therefore, on April 1, 2016, benidipine was switched to valsartan, and glibenclamide was switched to metformin. On April 2, the patient started to have headaches and palpitations, and on April 6, she visited our hospital. Plain computed tomography (CT) of the abdomen showed a $4-\mathrm{cm}$, isodense mass in the left adrenal gland (Fig. 1A), and the patient was hospitalized for further examination.

The patient's history was negative for smoking, alco- hol consumption, and allergies. Her family medical history was positive for many different malignant tumors, including her grandfather with gastric cancer, her mother with colon cancer and breast cancer, an older sister with endometrial cancer, and a younger sister of her mother with breast cancer. However, there was no family history of adrenal disease.

On physical examination, her height was $160 \mathrm{~cm}$, weight was $65.8 \mathrm{~kg}$, body mass index (BMI) was 25.7 $\mathrm{kg} / \mathrm{m}^{2}$, and she had hypertension with a blood pressure of $159 / 111 \mathrm{mmHg}$, tachycardia with a pulse rate of 126/ min, and a body temperature of $36.4^{\circ} \mathrm{C}$. The remainder of the physical examination was unremarkable except for hyperhidrosis. Laboratory tests showed no anemia or hepatorenal dysfunction, but there was an elevated inflammatory response with a white blood cell count of $15,400 / \mu \mathrm{L}$. The patient was also hyperglycemic, with a casual plasma glucose of $231 \mathrm{mg} / \mathrm{dL}$ and $\mathrm{HbAlc}$ of $8.5 \%$. Her total cholesterol was also high. A thyroid ultrasound examination and tumor markers were normal, with carcinoembryonic antigen (CEA) $0.7 \mathrm{ng} / \mathrm{mL}$ and calcitonin $<0.5 \mathrm{pg} / \mathrm{mL}$ (Table 1 ).

On hospital Day 1, insulin therapy for the hyperglycemia and antihypertensives for the hypertension were started. After starting nicardipine and doxazosin, propranolol was added for the tachycardia (Fig. 2). On Days 2 and 3, 
Table 1 General laboratory findings

\begin{tabular}{|c|c|c|}
\hline \multirow[t]{5}{*}{ Complete blood cell counts } & White blood cells $(/ \mu \mathrm{L})[3,290-7,810]$ & 15,400 \\
\hline & Red blood cells $(/ \mu \mathrm{L})\left[380 \times 10^{4}-496 \times 10^{4}\right]$ & $716 \times 10^{4}$ \\
\hline & Hemoglobin (g/dL) [11.8-15.0] & 16.7 \\
\hline & Hematocrit (\%) [35.2-44.5] & 51 \\
\hline & Platelets $(/ \mu \mathrm{L})\left[13.0 \times 10^{4}-36.0 \times 10^{4}\right]$ & $24.9 \times 10^{4}$ \\
\hline \multirow[t]{17}{*}{ Blood chemistry } & Total protein $(\mathrm{g} / \mathrm{dL})[6.5-8.2]$ & 7.5 \\
\hline & Albumin (g/dL) [3.9-4.9] & 4.1 \\
\hline & Blood urea nitrogen (mg/dL) [8-20] & 24 \\
\hline & Creatinine $(\mathrm{mg} / \mathrm{dL})[0.50-0.80]$ & 0.74 \\
\hline & Uric acid (mg/dL) [2.5-6.0] & 7.3 \\
\hline & Sodium $(\mathrm{mEq} / \mathrm{L})$ [135-147] & 139 \\
\hline & Chloride (mEq/L) [98-108] & 98 \\
\hline & Potassium $(\mathrm{mEq} / \mathrm{L})[3.5-5.0]$ & 4.3 \\
\hline & Aspartate aminotransferase (IU/L) [10-40] & 24 \\
\hline & Alanine aminotransferase (IU/L) [5-45] & 18 \\
\hline & Lactate dehydrogenase (IU/L) [120-240] & 293 \\
\hline & $C$ reactive protein $(\mathrm{mg} / \mathrm{dL})[<0.45]$ & 0.55 \\
\hline & Casual Glucose (mg/dL) & 231 \\
\hline & Hemoglobin A1c (\%) [4.7-6.2] & 8.5 \\
\hline & Total cholesterol (mg/dL) [120-220] & 334 \\
\hline & Low density lipoprotein $(\mathrm{mg} / \mathrm{dL})[70-140]$ & 188 \\
\hline & Triglycerides (mg/dL) [30-150] & 131 \\
\hline \multirow[t]{2}{*}{ Tumor markers } & Carcinoembryonic antigen $(\mathrm{ng} / \mathrm{mL})[<5.0]$ & 0.7 \\
\hline & Calcitonin $(\mathrm{pg} / \mathrm{mL})[<54.0]$ & $<0.5$ \\
\hline
\end{tabular}

[ ]: normal reference value

the serum and urinary catecholamine levels were markedly elevated. Plasma adrenocorticotrophic hormone (ACTH) and cortisol levels were also high (Table 2). The patient spontaneously experienced episodic left flank pain on Day 4 without any preceding events. Immediately after this episode, blood pressure and glucose levels were decreased. Her left flank pain tended to subside without any analgesics. Antihypertensive and insulin therapies were no longer required (Fig. 2).

Plain CT at that time showed heterogeneous high density areas in the left adrenal mass (Fig. 1B). Shock occurred on Day 5 , with a systolic blood pressure $\leq 90 \mathrm{mmHg}$, and a large quantity of intravenous fluids was temporarily required to maintain blood pressure (Fig. 2). The patient had an elevated inflammatory response with a fever of about $38^{\circ} \mathrm{C}$ (Fig. 2). T2-weighted magnetic resonance imaging (MRI) on Day 6 showed high signal intensity within a tumor with an indistinct border in the left adrenal gland (Fig. 1C).

On Day 7, 3 meta-iodobenzylguanidine (MIBG) scintigraphy showed no abnormal uptake (Fig. 1D). On Day 8 , contrast CT showed low density areas within the left adrenal tumor, contrast enhancement of the tumor margins, and tumor capsule rupture (Fig. 1E). The left flank pain continued to subside, and the patient was now afebrile with a tendency for a decreased inflammatory response (Fig. 2). Moreover, on Day 9 and later, the serum and urinary catecholamine levels were markedly decreased, with a return to within near-normal reference values (Table 2). On Day 13 and later, blood pressure, 


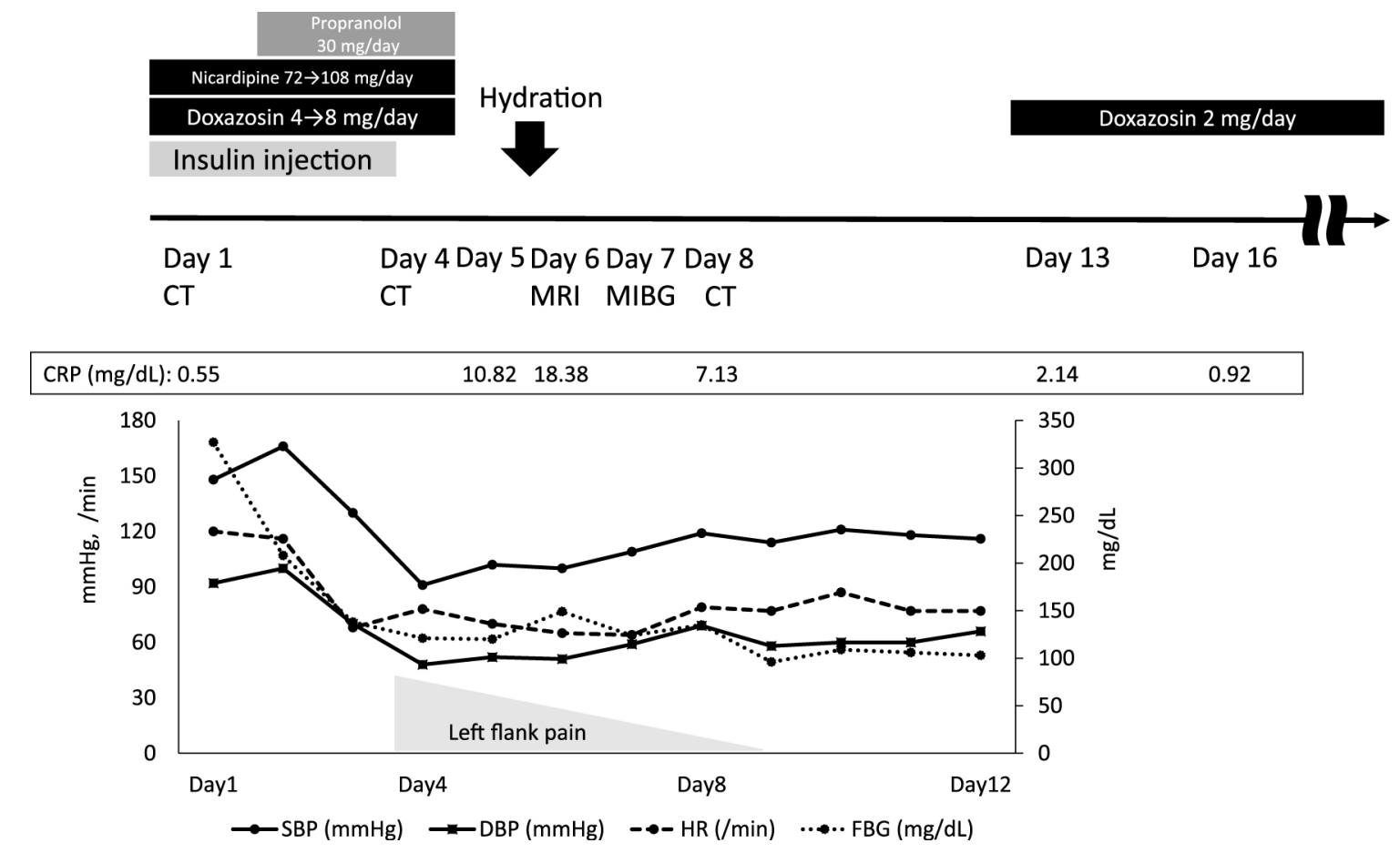

Fig. 2 Course after hospital admission

Drug therapy was started after admission to control blood pressure, pulse rate, and glucose levels. On Day 4 after the onset of left flank pain, antihypertensive and insulin therapies were no longer necessary. Shock occurred on day 5 with a systolic blood pressure $\leq 90 \mathrm{mmHg}$, and a large quantity of intravenous fluids was temporarily required to maintain blood pressure. There was also an elevated inflammatory response. Starting on Day 8, the left flank pain subsided, and the inflammatory response decreased. On Day 13 and later, blood pressure, blood glucose, and pulse rate were normal with oral doxazosin $2 \mathrm{mg}$ alone, and the inflammatory response continued to subside. CT, Computed tomography; HU, Hounsfield units; MRI, Magnetic resonance imaging; MIBG, 3 meta-iodobenzylguanidine; CRP, C-reactive protein; SBP, Systolic blood pressure; DBP, Diastolic blood pressure; HR, Heart rate; FBG, Fasting blood glucose.

blood glucose, and pulse rate were normal with oral doxazosin $2 \mathrm{mg}$ /day alone. The inflammatory response continued to subside (Fig. 2).

A left adrenal tumor resection was electively performed on Day 49. The capsule of the resected tumor was ruptured (Fig. 3A, 3B). Hematoxylin-eosin (HE) staining showed widespread hemorrhagic necrosis in the center of the tumor (Fig. 3C). The viable area was located on the periphery of the tumor (Fig. 3D). Viable cell components in the tumor margins, including cells that had abundant eosinophilic cytoplasm, were arranged in an alveolar pattern (a Zellballen pattern) with rich vascularities. The histopathologic diagnosis was pheochromocytoma (Fig. 3C, 3D). Immunostaining of the tumor cells was positive for chromogranin A (Fig. 3E), but with only a Ki67-positive rate of $<2 \%$ (Fig. 3F).

Since surgery, the serum catecholamine levels and $\mathrm{HbAlc}$ have returned to within normal reference values.
There have been no further symptoms, and blood pressure at home has been stable in the range of about 120$130 / 70-80 \mathrm{mmHg}$ with oral amlodipine $5 \mathrm{mg} /$ day.

\section{Discussion}

The present patient had a left adrenal mass on the admission CT, and, based on markedly elevated catecholamine levels, headaches, hyperhidrosis, tachycardia, hypertension, and hyperglycemia, a pheochromocytoma was suspected. Hospital management of blood pressure, pulse rate, and glucose levels included antihypertensives and insulin. However, after the onset of left flank pain (Day 4), blood pressure and glucose levels decreased abruptly, and antihypertensive and insulin therapies were gradually discontinued. This was associated with a decrease in catecholamine secretion after the development of left flank pain on Day 4. 
Table 2 Hormone profiles

\begin{tabular}{|c|c|c|c|c|c|c|c|}
\hline & & Day 2 & Day 3 & Day 9 & Day 13 & Day 17 & Day 25 \\
\hline \multirow[t]{7}{*}{ Plasma } & Adrenocorticotrophic hormone $(\mathrm{pg} / \mathrm{mL})$ [7.2-63.3] & 77.8 & & & 13.8 & & 16.4 \\
\hline & Cortisol ( $\mu \mathrm{g} / \mathrm{dL})[4.5-21.1]$ & 36.6 & & & 8.2 & & 9.6 \\
\hline & Renin activity (ng/mL/h) [0.3-2.9] & 3.3 & & & $<0.2$ & & $<0.2$ \\
\hline & Aldosterone (pg/mL) [29.9-158.8] & 114.3 & & & $<25$ & & 69.4 \\
\hline & Adrenalin $(\mathrm{pg} / \mathrm{mL})[0-100]$ & 4,721 & & & 43 & & 44 \\
\hline & Noradrenalin $(\mathrm{pg} / \mathrm{mL})[140-450]$ & 20,458 & & & 645 & & 212 \\
\hline & Dopamine $(\mathrm{pg} / \mathrm{mL})[0-20]$ & 256 & & & 16 & & $<10$ \\
\hline \multirow[t]{7}{*}{ Urine } & Homovanillic acid (mg/day) [1-10.3] & & 5.6 & 2.8 & & 5.1 & \\
\hline & Vanilmandelic acid (mg/day) [1.1-7.2] & & 48.1 & 6.7 & & 4.2 & \\
\hline & Adrenalin ( $\mu \mathrm{g} /$ day) [3.4-26.9] & & $2,334.1$ & 32.9 & & 16.7 & \\
\hline & Noradrenalin ( $\mu \mathrm{g} /$ day) $[48.6-168.4]$ & & $8,612.8$ & 518 & & 261.5 & \\
\hline & Dopamine ( $\mu \mathrm{g} /$ day) [365-961.5] & & 1,629 & 468.3 & & 747.3 & \\
\hline & Metanephrine (mg/day) [0.04-0.19] & & 5.76 & 0.22 & & 0.11 & \\
\hline & Normetanephrine (mg/day) [0.09-0.33] & & 6.84 & 0.7 & & 0.38 & \\
\hline
\end{tabular}

[ ]: normal reference value

MIBG scintigraphy and measurement of serum and urinary catecholamine levels are useful for functional evaluation of pheochromocytomas [4]. MIBG scintigraphy in the present patient on Day 7 showed no abnormal uptake, and there was also a marked decrease in serum and urinary catecholamine levels. These findings suggested a substantial loss of tumor cell function.

Plain CT on admission showed isodensity within the left adrenal tumor, but after the onset of left flank pain (Day 4), plain CT showed high density areas within the tumor. Hemorrhage within a few days on plain CT of the adrenal glands can be identified by high-density areas (50-90 HU) [5]. The high-density areas in the left adrenal tumor on plain CT on Day 4 were suspicious of intratumoral hemorrhage. The left flank pain that occurred on Day 4 was also suspicious for left adrenal intratumoral hemorrhage.

T2-weighted MRI on Day 6 showed a tumor with an indistinct border and mixed heterogeneous high and low signal areas within the tumor. Adrenal hemorrhage on MRI is depicted as a low signal area because of blood cell components (oxyhemoglobin) at one week after hemorrhage, but about one week later, serum components lead to depiction as a high signal area [6]. The heterogeneity within the tumor on T2-weighted MRI on Day 6 suggests temporal changes after adrenal hemor- rhage. The indistinct tumor border was probably due to leakage of blood cells and serum components associated with intratumoral hemorrhage and capsule rupture.

Contrast CT on Day 8 showed low density areas within the tumor and increased contrast enhancement of the tumor margins. Contrast enhancement on CT can depict the cellular components of pheochromocytomas and the adrenal capsule [7]. Moreover, tumor components just after hemorrhage of adrenal tumors can be identified as low-density areas on CT [5]. The lowdensity areas within the tumor seen on contrast CT on Day 8 were suspicious of intratumoral hemorrhage. In addition, contrast enhancement of the tumor margins seemed to be consistent with a capsule and/or residual viable tumor cells.

Better outcomes with elective rather than emergency surgery have been reported in patients with spontaneous rupture of a pheochromocytoma [1]. In this report, a high frequency of preoperative shock and absence of an accurate diagnosis in many such patients were poor prognostic factors. Our approach to a suspected pheochromocytoma rupture in the present case was, first, hemodynamic management, followed by elective left adrenal tumor resection that resulted in a good postoperative course.

Macroscopic examination of the resected tumor in the 

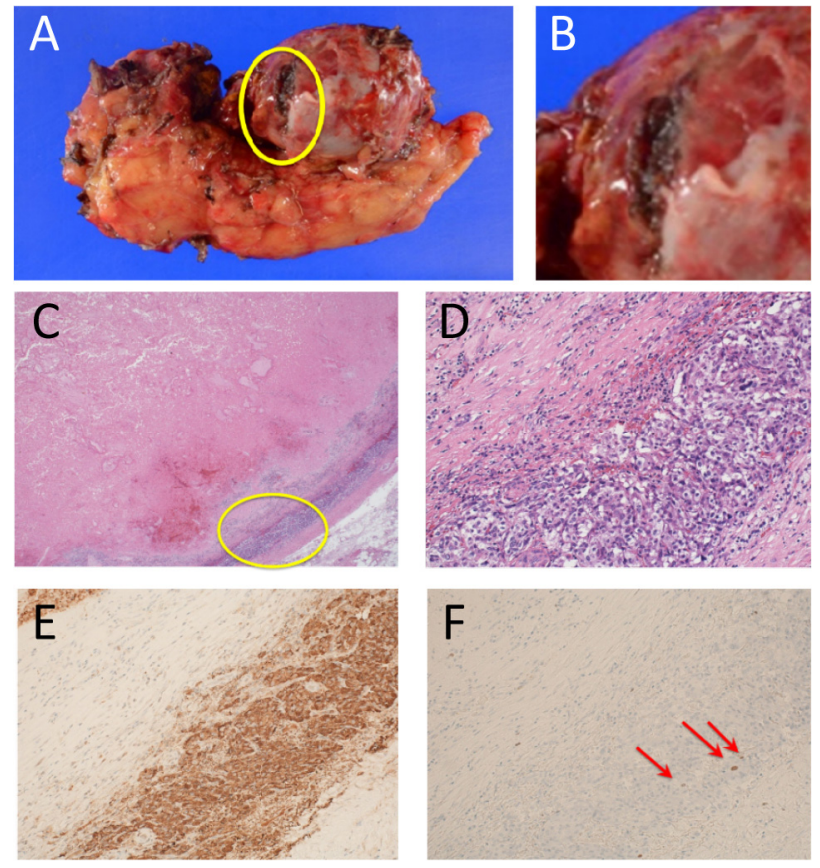

Fig. 3 Macroscopy and histopathology of the resected tumor

A: Macroscopic examination of the resected tumor. B is a magnified view of the area surrounded by the yellow oval in A. B: Magnified macroscopic view of the resected tumor shows a fissure in the tumor surface. C: Histopathology/HE staining $\times 20$. D is a magnified view of the area surrounded by the yellow oval in C. D: Histopathology/HE staining $\times 200$. E: Histopathology with immunostaining for anti-chromogranin A antibody $\times 200$. F: Histopathology with immunostaining for anti-Ki67 antibody $\times 200$. Red arrows show positive staining.

present patient showed capsule rupture. Histopathology showed hemorrhagic necrosis of the tumor with viable cells localized in the margins. Staining for chromogranin A was positive, but the Ki67-positive rate was $\leq 2 \%$. Chromogranin A is a neuroendocrine cell marker that is positive in pheochromocytomas [8]. Ki67 is a tumor proliferation marker, and the Ki67-positive rate of $\leq 2 \%$ indicates low proliferation [9]. Therefore, the viable cells were consistent with a neuroendocrine tumor (pheochromocytoma), and histopathology suggested pheochromocytoma rupture and hemorrhagic necrosis. Based on the above findings, we presumed that tumor cell necrosis resulted in a marked reduction in catecholamine levels.

The cause of intratumoral hemorrhage and rupture was probably increased intratumoral pressure. A massive release of catecholamines from pheochromocytoma into the circulation probably accelerates the progression of tumor infarction, resulting in the extensive necrosis of adrenal pheochromocytoma [10]. Acute infarction and subsequent necrosis decrease the release of catecholamines from the tumor. Vasodilation in the tumor, due to the decreased release of catecholamines, results in increased blood flow into the tumor and high intratumoral pressure. High intratumoral pressure in the capsulated tumor may also result in a tear in the capsule and the rupture of pheochromocytoma [11]. In addition, because blood outflow vessels in the adrenal glands are much narrower than inflow vessels, vasodilation and vascular smooth muscle relaxation cause adrenal blood congestion, thus raising adrenal gland pressure [12]. The anatomical characteristics of the adrenal gland may also have contributed to an increase in intratumoral pressure.

Moreover, alpha-blockers have been reported to cause tumor rupture in pheochromocytoma [13]. According to that report, tumor vessel contraction due to catecholamine effects, followed by vasodilation due to an alphablocker, leads to increased blood flow to tumors. This can result in tumor rupture due to an increase in intratumoral pressure. Antihypertensive drugs in the present patient had been switched before hospitalization. This switch in medications before hospital admission may have caused an increase in blood pressure. Subsequently, the patient had a fall in blood pressure with nicardipine and doxazosin during hospitalization, and then on Day 4, she had clinical findings suggesting tumor rupture. Therefore, the possibility that administration of alphablockers and these changes in blood pressure were associated with an increase in intratumoral pressure, hemorrhage, and rupture cannot be excluded. In patients with pheochromocytoma, unstable systemic blood pressure and hemodynamics within the tumor, due to episodic release of catecholamines and/or use of antihypertensives seem to be associated with tumor infarction and rupture. In such patients, abdominal CT scanning is recommended when the patients display abdominal pain.

Most previously reported cases of spontaneous large necrosis of adrenal pheochromocytoma were shown to be benign, but malignant cases have also been reported [14]. In the present case, since the tumor remained to be large in size after the suspected rupture (Fig. 1E), and urine noradrenalin and normetanephrine levels were still modestly elevated (Table 2), surgical removal was performed. As a result, histopathological findings suggested low risk of malignancy. The patient displayed no signs of recurrence during more than two years of postoperative follow-up period. Since recurrence of pheochromocytoma could occur even decades after surgery [15], further 
careful follow-up is mandatory.

Pheochromocytoma occasionally produces interleukin 6 (IL-6), and fever and increased CRP levels are characteristics of such pheochromocytoma [16]. The present case was afebrile, and CRP level was modestly elevated on admission. After the suspected necrosis and rupture of the tumor, CRP level was increased (Fig. 2). Therefore, increased CRP levels during the course seem to be due to the necrosis and rupture of the tumor.

In conclusion, the present patient displayed remarkable changes in imaging findings and hormone levels before and after pheochromocytoma rupture. Following elective surgery for tumor resection, pheochromocytoma rupture and hemorrhagic necrosis were confirmed. In patients with a suspected pheochromocytoma rupture, adequate hemodynamic management is mandatory, and if the remission of pheochromocytoma function is observed, safe elective surgery can be performed for tumor resection.

\section{Acknowledgements}

The submission of this case report is approved by the local ethics committee (F2018C02), and the written informed consent is obtained from the patient.

\section{Disclosure}

None of the authors have any potential conflicts of interest associated with this research.

\section{References}

1. Kobayashi T, Iwai A, Takahashi R, Ide Y, Nishizawa K, et al. (2005) Spontaneous rupture of adrenal pheochromocytoma: review and analysis of prognostic factors. J Surg Oncol 90: 31-35.

2. Atuk NO, Teja K, Mondzelewski JP, Turner SM, Selden RF (1977) Avascular necrosis of pheochromocytoma followed by spontaneous remission. Arch Intern Med 137: 1073-1075.

3. Maruyama M, Sato H, Yagame M, Shoji S, Terachi T, et al. (2008) Spontaneous rupture of pheochromocytoma and its clinical features: a case report. Tokai J Exp Clin Med 33: 110-115.

4. Lynn MD, Shapiro B, Sisson JC, Beierwaltes WH, Meyers LJ, et al. (1985) Pheochromocytoma and the normal adrenal medulla: improved visualization with I-123 MIBG scintigraphy. Radiology 155: 789-792.

5. Dunnick NR (1990) Adrenal imaging: current status. AJR Am J Roentgenol 154: 927-936.

6. Rubin JI, Gomori JM, Grossman RI, Gefter WB, Kressel HY (1987) High-field MR imaging of extracranial hematomas. AJR Am J Roentgenol 148: 813-817.

7. Blake MA, Kalra MK, Maher MM, Sahani DV, Sweeney AT, et al. (2004) Pheochromocytoma: an imaging chameleon. Radiographics 24 Suppl 1: S87-S99.

8. Millar AD, Masek-Hammerman K, Dalecki K, Mansfield KG, Westmoreland SV (2009) Histologic and immunohistochemical characterization of pheochromocytoma in six cotton-top tamarins (Saguinus Oedipus). Vet Pathol 46: 1221-1229.

9. Thompson LD (2002) Pheochromocytoma of the Adrenal gland Scaled Score (PASS) to separate benign from malignant neoplasms: a clinicopathologic and immunophenotypic study of 100 cases. Am J Surg Pathol 26: 551-566.

10. Ohara N, Uemura Y, Mezaki N, Kimura K, Kaneko M, et al. (2016) Histopathological analysis of spontaneous large necrosis of adrenal pheochromocytoma manifested as acute attacks of alternating hypertension and hypotension: a case report. J Med Case Rep 10: 279-284.

11. Hanna JS, Spencer PJ, Savopoulou C, Kwasnik E, Askari R (2011) Spontaneous adrenal pheochromocytoma rupture complicated by intraperitoneal hemorrhage and shock. World J Emerg Surg 6: 27-33.

12. Rao RH, Vagnucci AH, Amico JA (1989) Bilateral massive adrenal hemorrhage: early recognition and treatment. Ann Intern Med 110: 227-235.

13. Van Way CW 3rd, Faraci RP, Cleveland HC, Foster JF, Scott HW Jr (1976) Hemorrhagic necrosis of pheochromocytoma associated with phentolamine administration. Ann Surg 184: 26-30.

14. Nyman D, Wahlberg P (1970) Necrotic phaeochromocytoma with gastric haemorrhage, shock, and uncommonly high catecholamine excretion. Acta Med Scand 187: 381383.

15. Thai E, Gnetti L, Gilli A, Caruana P, Dalla Valle R, et al. (2015) Very late recurrence of an apparently benign pheochromocytoma. J Cancer Res Ther 11: 1036.

16. Kang JM, Lee WJ, Kim WB, Kim TY, Koh JM, et al. (2005) Systemic inflammatory syndrome and hepatic inflammatory cell infiltration caused by an interleukin-6 producing pheochromocytoma. Endocr J 52: 193-198. 